\title{
THE PRICING MODEL OF THE BARRIER OPTION UNDER THE TRINOMIAL TREE
}

\section{ZHIKAI YAN}

Department of Mathematics

Jinan University

Guangzhou, Guangdong 510632

P. R. China

e-mail: 18271672419@163.com

\begin{abstract}
The establishment of the trinomial tree model provides a convenient way to pricing the options, comparing to the binary tree, it has the advantage of higher accuracy and feasibility. In this paper, based on the trinomial tree model, we are researching the pricing model of the barrier options, we can obtain a new model to pricing the barrier options, and this model can be a valid way to pricing the barrier options.
\end{abstract}

\section{Preliminary Knowledge}

\subsection{The introduction of the barrier options}

The option is the core tool of the risk management, it's an objective option, which can give the right to buyers to buy or sold a certain number of initiative assets according to the promised price. The buyers of the options only have the right but no obligation to meet the engagement, the sellers must obey the buyers' right. With the rapidly progress of the 2010 Mathematics Subject Classification: 97M30, 97M4.

Keywords and phrases: the barrier option, the trinomial tree, the price of option.

Received April 14, 2018; Revised July 5, 2018

(ㄷ) 2018 Scientific Advances Publishers 
option market, in order to satisfy the different investment demands of different investors, the new type of option has been invented. Among all the exotic options, the barrier option is the earliest emerged one.

The barrier option is actually a sort of conditional options, whether it is valid depends on whether the price of the underlying asset has touched the barrier value set before, therefore it is also called the path-related option. In recent years, the path-related option is becoming more and more popular, so the barrier option has become a very widely used option type in the financial market. The barrier options can be classified into two main kinds: knock-in options and knock-out options. When the price of the underlying assets arrives at the specific values, the options become valid, they are called knock-in options. When the price of the underlying assets arrives at the specific values, the options become invalid, they are called knock-out options. During a valid period, if a option has been knocked out or hasn't been knocked in, the option becomes invalid, the holder of the option will be paid to amount of cash as compensation. As for knock out option, when the initial price of the underlying asset is higher than the barrier value, it is called down and out option. On the contrary, when the price is lower than the barrier value, it is called up and out option. Similarly, according to the size of the price of the underlying asset and the barrier value, we can define the down and in option as well as up and in option. Moreover, the option also can be classified into call option and put option, so the barrier option can be classified into 8 kinds: the up and in call option, the up and in put option, the up and out call option, the up and out put option, the down and in call option, the down and in put option, the down and out call option, and the down and out put option. The value of the barrier options can be written as:

The up and in call option $\left(S_{T}-K\right)^{+} \circ I_{\left(\tau_{H} \leq T\right)}, H>K$;

where $\tau_{H}=\inf \left\{t>0, S_{t}=H\right\}$, 
$H$ means the price of the barrier value, $I$ is an indicative function, when conditions are suitable, it values 1 , otherwise it values 0 .

\subsection{The introduction of the trinomial tree model}

\subsubsection{The fundamental assumption}

Theoretically, before establishing the trinomial tree model, we assume that:

(1) All the resource information of investors will be shared.

(2) There is no friction in the market, that means no transaction cost and tax in the trade.

(3) The financial market is complete, so it means no arbitrage chance.

(4) The expected yield rate $\mu$ and the volatility rate $\sigma$ of the underlying asset are both constant, what's more, the risk-free interest rate $r$ is equal to the expected yield rate.

(5) The underlying asset obeys the geometric Brownian motion $\frac{d S(t)}{S(t)}=\mu d t+\sigma d z(t)$ in the period of $[0, T], z(t)$ is a standard Brownian motion.

\subsubsection{The discretizaton of the underlying asset}

Divide the time interval $[0, T]$ into $n$ equal division, the length of every time interval is $\Delta t=\frac{T}{n}\left(0=t_{0}<t_{1}<t_{2}<\cdots<t_{n}=T\right), T$ is the expiration time. During the $\left[t_{i}, t_{i}+\Delta t\right]$, the variation of the underlying asset obeys the geometric Brownian motion: $\frac{\Delta S}{S}=\mu \Delta t+\sigma\left(z\left(t_{i}+\Delta t\right)-z\left(t_{i}\right)\right)$. According to the ITO integral, we can obtain that: $S(t)=S\left(t_{i}\right)$ $e^{\sigma\left(z\left(t_{i}+\Delta t\right)-z\left(t_{i}\right)\right)+\left(\mu-\frac{\sigma^{2}}{2}\right)\left(t-t_{i}\right)}$. For $S(t)$ obeys the ITO process, $S^{2}(t), S^{3}(t)$ 
are functions regard to $S(t)$ and $t$. So $S^{2}(t), S^{3}(t)$ also obey the ITO process, under the continuous condition:

$$
\begin{aligned}
& S^{2}(t)=S^{2}\left(t_{i}\right) e^{2 \sigma\left(z(t)-z\left(t_{i}\right)+\left(2 \mu-\sigma^{2}\right)\left(t-t_{i}\right)\right.}, \\
& S^{3}(t)=S^{3}\left(t_{i}\right) e^{3 \sigma\left(z(t)-z\left(t_{i}\right)+\left(3 \mu-\frac{3}{2} \sigma^{2}\right)\left(t-t_{i}\right)\right.},
\end{aligned}
$$

$z(t)$ is a standard Brownian motion, so $E[z(t)]=0$, $\operatorname{Var}[z(t)]=1$, we can obtain that:

$$
E\left[e^{\sigma z(t)-\frac{\sigma^{2}}{2}}\right]=e^{-\frac{\sigma^{2}}{2}} E\left[e^{\sigma z(t)}\right]=e^{-\frac{\sigma^{2}}{2}} \frac{1}{\sqrt{2 \pi}} \int_{-\infty}^{+\infty} e^{\frac{\sigma^{2}}{2}} e^{-\frac{(x-\sigma)^{2}}{2}} d x=1
$$

Similarly, $E\left[e^{2 \sigma z(t)-2 \sigma^{2}}\right]=1, E\left[e^{3 \sigma z(t)-\frac{9}{2} \sigma^{2}}\right]=1$, what's more,

$$
\begin{gathered}
\left.E[S(t)]=E\left[S\left(t_{i}\right)\right] \exp \left(\sigma\left(z(t)-z\left(t_{i}\right)\right)+\left(\mu-\frac{\sigma^{2}}{2}\right)\left(t-t_{i}\right)\right)\right]=S\left(t_{i}\right) e^{\mu\left(t-t_{i}\right)}, \quad(1) \\
\left.E\left[S^{2}(t)\right]=E\left[S^{2}\left(t_{i}\right)\right] \exp \left(2 \sigma\left(z(t)-z\left(t_{i}\right)\right)+\left(2 \mu-\sigma^{2}\right)\left(t-t_{i}\right)\right)\right]=S^{2}\left(t_{i}\right) e^{\left(2 \mu+\sigma^{2}\right)\left(t-t_{i}\right)},
\end{gathered}
$$

$$
E\left[S^{3}(t)\right]=E\left[S^{3}\left(t_{i}\right) \exp \left(3 \sigma\left(z(t)-z\left(t_{i}\right)\right)+\left(3 \mu-\frac{3}{2} \sigma^{2}\right)\left(t-t_{i}\right)\right)\right]=S^{3}\left(t_{i}\right) e^{\left(3 \mu+3 \sigma^{2}\right)\left(t-t_{i}\right)} .
$$

\subsubsection{The establishment of the trinomial tree model}

Assume that during $\left[t_{i}, t_{i}+\Delta t\right]$, the price of the underlying asset will variate in three ways, illustrated in Figure 1, $u$ and $d$ stand for the range of go up and go down of the underlying assets respectively, and they must satisfy: $u>1, d<1, u d=1$. 


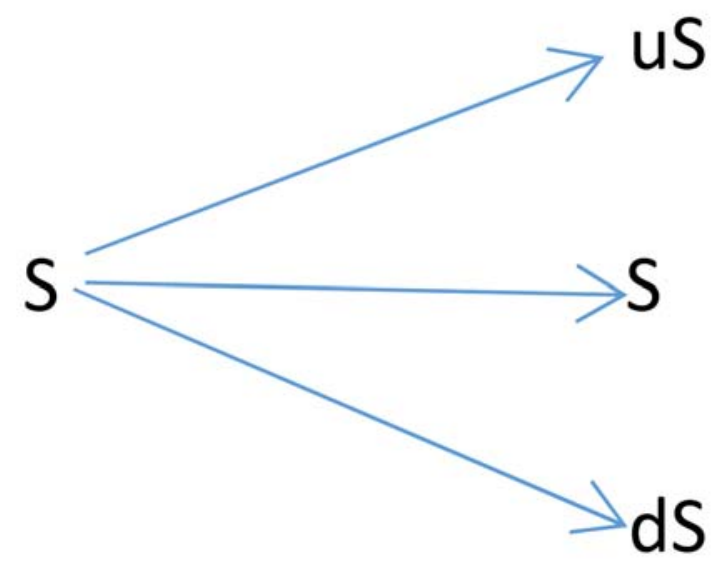

Figure 1. The three stage of the underlying asset.

The probability to go up named $P_{u}$, the probability to go down named $P_{d}$, the probability to remain unchanged named $P_{m}$.

According to the definition of the expectation, we can obtain that:

$$
\begin{gathered}
E[S(t)]=P_{u} u S\left(t_{i}\right)+P_{m} S\left(t_{i}\right)+P_{d} d S\left(t_{i}\right), \\
E\left[S^{2}(t)\right]=P_{u}\left(u S\left(t_{i}\right)\right)^{2}+P_{m} S^{2}\left(t_{i}\right)+P_{d}\left(d S\left(t_{i}\right)\right)^{2}, \\
E\left[S^{3}(t)\right]=P_{u}\left(u S\left(t_{i}\right)\right)^{3}+P_{m} S^{3}\left(t_{i}\right)+P_{d}\left(d S\left(t_{i}\right)\right)^{3} .
\end{gathered}
$$

Combine (1), (2), and (3), we can obtain a equation set:

$$
\left\{\begin{array}{l}
P_{u}+P_{d}+P_{m}=1, \\
P_{u} u+P_{m}+P_{d} d=e^{\mu \Delta t}, \\
P_{u} u^{2}+P_{m}+P_{d} d^{2}=e^{\left(2 \mu+\sigma^{2}\right) \Delta t}, \\
P_{u} u^{3}+P_{m}+P_{d} d^{3}=e^{\left(3 \mu+3 \sigma^{2}\right) \Delta t}, \\
u d=1 .
\end{array}\right.
$$


According to the three equations mentioned above, we can construct a matrix to solve this equation set

$$
\left[\begin{array}{cccc}
1 & 1 & 1 & 1 \\
u & 1 & d & e^{\mu \Delta t} \\
u^{2} & 1 & d^{2} & e^{\left(2 \mu+\sigma^{2}\right) \Delta t}
\end{array}\right] .
$$

By Cramer's rule we can solve this equation set, the result is:

$$
\left\{\begin{array}{l}
P_{u}=\frac{(1+d) e^{\mu \Delta t}-e^{\left(2 \mu+\sigma^{2}\right) \Delta t}-d}{(d-u)(u-1)} \\
P_{m}=\frac{(u+d) e^{\mu \Delta t}-e^{\left(2 \mu+\sigma^{2}\right) \Delta t}-1}{(1-d)(u-1)} \\
P_{d}=\frac{(1+u) e^{\mu \Delta t}-e^{\left(2 \mu+\sigma^{2}\right) \Delta t}-u}{(d-u)(1-d)}
\end{array}\right.
$$

Substitute $P_{u}, P_{m}, P_{d}$ into the 4 th equation of the equation set, and combined with the 5 th equation we can obtain:

$$
\begin{gathered}
u=M+\sqrt{M^{2}-1}, d=M-\sqrt{M^{2}-1}, \\
M=\frac{e^{\mu \Delta t}+e^{\left(3 \mu+3 \sigma^{2}\right) \Delta t}-e^{\left(2 \mu+\sigma^{2}\right)}-1}{2\left[e^{\left(2 \mu+\sigma^{2}\right) \Delta t}-e^{\mu \Delta t}\right]} .
\end{gathered}
$$

By using Taylor expansion, we can simplify the $P_{u}, P_{m}, P_{d}, u, d$ just like:

$$
\begin{aligned}
& u=e^{\sigma \sqrt{3 \Delta t}}=1+\sigma \sqrt{3 \Delta t}+\frac{3}{2} \sigma^{2} \Delta t+o(\Delta t), \\
& d=e^{-\sigma \sqrt{3 \Delta t}}=1-\sigma \sqrt{3 \Delta t}+\frac{3}{2} \sigma^{2} \Delta t+o(\Delta t), \\
& P_{u}=\frac{1}{6}+\left(\mu-\frac{\sigma^{2}}{2}\right) \sqrt{\frac{\Delta t}{12 \sigma^{2}}}+o(\sqrt{\Delta t}),
\end{aligned}
$$




$$
\begin{aligned}
& P_{m}=\frac{2}{3}+o(\sqrt{\Delta t}), \\
& P_{d}=\frac{1}{6}-\left(\mu-\frac{\sigma^{2}}{2}\right) \sqrt{\frac{\Delta t}{12 \sigma^{2}}}+o(\sqrt{\Delta t}) .
\end{aligned}
$$

Resemble to the binary tree model, use the forward inductive method to construct the trinomial tree model, use backward retrodict method to pricing the barrier options. That is to say, the price of option at the moment of $i \Delta t$ can be discounted by the price in the moment of $(i+1) \Delta t$, it can be expressed as:

$$
C(S, i \Delta t)=e^{-r \Delta t}\left[P_{u} C(u S,(i+1) \Delta t)+P_{m} C(S,(i+1) \Delta t)+P_{d} C(d S,(i+1) \Delta t)\right] .
$$

In the (7), $C(S, i \Delta t)$ means the price of the option at the time of $i \Delta t$, $C(u S,(i+1) \Delta t), C(S,(i+1) \Delta t), \quad$ and $C(d S,(i+1) \Delta t)$ stand for the condition of go up, stay unchanged, and go down of the underlying assets at the time of $(i+1) \Delta t$.

\section{The Pricing Model of the Barrier Option Under the Trinomial Tree Model}

Now, without loss of generality, we take the up and in call barrier option into consideration, the barrier value $H$ has been set advanced. The underlying asset whose exercise price is $K$ has a expiration period of $T$, what's more, at the moment of $T$, the price of the underlying asset is $S_{T}$. We can use $C(t)$ to express the value of the barrier option during $[0, T]$, use $S(t)$ to express the value of the underlying asset. For the up and in barrier option, only when the price of the underlying asset goes above and touches the barrier value set before, can the option come into validity. Otherwise, the option is invalid. 
Assume that there exist $n$ satisfies $\Delta t=\frac{T}{n}\left(0=t_{0}<t_{1}<\cdots<t_{n}\right)$. Figure 2 and Figure 3 can show us the variations of the underlying asset and the option, Figure 4 shows the variations of the price the up and in barrier option.
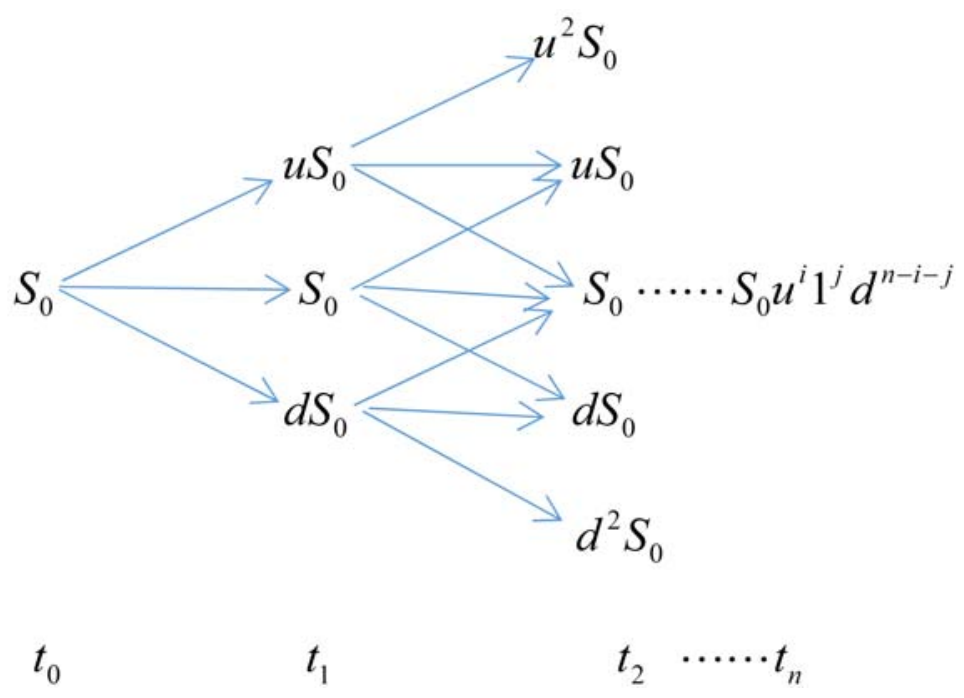

Figure 2. The variations of the underlying asset. 

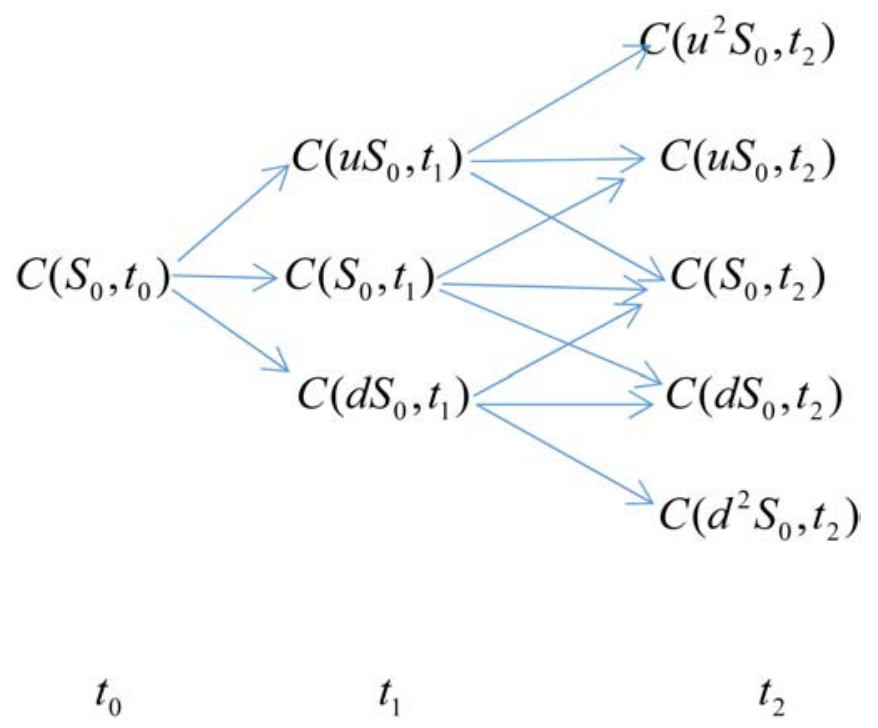

Figure 3. The variations of the option.

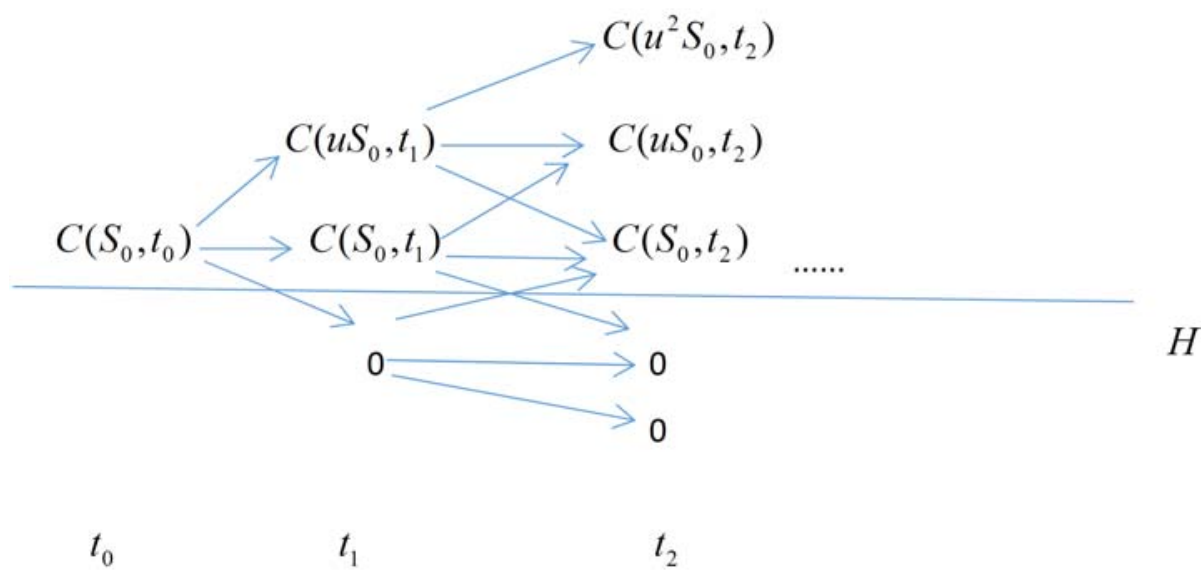

Figure 4. The trinomial tree model of the up and in call option. 
According to the Figures above, combined with the value formula of the option, the value of the up and in call option at the moment of $T$ is:

$$
\left(S_{T}-K\right)^{+} \circ I_{\left(\tau_{H}<T\right)}, H>K
$$

Based on (7), by the mathematical induction method, we can infer the pricing model under the trinomial tree model of the up and in call option is:

$$
\begin{array}{r}
C\left(S_{0}, 0\right)=e^{-r T} \sum_{i=0}^{n} \sum_{j=n-i}^{n} \frac{n !}{i ! j !(n-i-j) !} P_{u}^{i} P_{m}^{j} P_{d}^{n-i-j}\left[\left(S_{T}-K\right)^{+} \circ I_{\left(\tau_{H} \leq T\right)}\right. \\
\left.-S_{0} u^{i} 1^{j} d^{n-i-j}\right], H>K,
\end{array}
$$

where $\tau_{H}=\inf \left\{t>0, S_{t}=H\right\}$,

$H$ means the price of the barrier value, $I$ is an indicative function, when conditions are suitable, it values 1 , otherwise it values 0 .

\section{The Pricing Model of the Other Barrier Options}

Similarly, we can infer the other 7 kinds of barrier option pricing model is as follow:

The up and in put option:

$$
\begin{aligned}
C\left(S_{0}, 0\right)=e^{-r T} \sum_{i=0}^{n} \sum_{j=n-i}^{n} \frac{n !}{i ! j !(n-i-j) !} & P_{u}^{i} P_{m}^{j} P_{d}^{n-i-j}\left[S_{0} u^{i} 1^{j} d^{n-i-j}\right. \\
& \left.-\left(K-S_{T}\right)^{+} \circ I_{\left(\tau_{H} \leq T\right)}\right], H>K .
\end{aligned}
$$


The up and out call option:

$$
\begin{array}{r}
C\left(S_{0}, 0\right)=e^{-r T} \sum_{i=0}^{n} \sum_{j=n-i}^{n} \frac{n !}{i ! j !(n-i-j) !} P_{u}^{i} P_{m}^{j} P_{d}^{n-i-j}\left[\left(S_{T}-K\right)^{+} \circ I_{\left(\tau_{H}>T\right)}\right. \\
\left.-S_{0} u^{i} 1^{j} d^{n-i-j}\right], H>K .
\end{array}
$$

The up and out put option:

$$
\begin{aligned}
C\left(S_{0}, 0\right)=e^{-r T} \sum_{i=0}^{n} \sum_{j=n-i}^{n} \frac{n !}{i ! j !(n-i-j) !} & P_{u}^{i} P_{m}^{j} P_{d}^{n-i-j}\left[S_{0} u^{i} 1^{j} d^{n-i-j}\right. \\
& \left.-\left(S_{T}-K\right)^{+} \circ I_{\left(\tau_{H}>T\right)}\right], H>K .
\end{aligned}
$$

The down and in call option:

$$
\begin{array}{r}
C\left(S_{0}, 0\right)=e^{-r T} \sum_{i=0}^{n} \sum_{i=n-i}^{n} \frac{n !}{i ! j !(n-i-j) !} P_{u}^{i} P_{m}^{j} P_{d}^{n-i-j}\left[\left(S_{T}-K\right)^{+} \circ I_{\left(\tau_{H} \leq T\right)}\right. \\
\left.-S_{0} u^{i} 1^{j} d^{n-i-j}\right], H<K .
\end{array}
$$

The down and in put option:

$$
\begin{aligned}
C\left(S_{0}, 0\right)=e^{-r T} \sum_{i=0}^{n} \sum_{j=n-i}^{n} \frac{n !}{i ! j !(n-i-j) !} & P_{u}^{i} P_{m}^{j} P_{d}^{n-i-j}\left[S_{0} u^{i} 1^{j} d^{n-i-j}\right. \\
& \left.-\left(K-S_{T}\right)^{+} \circ I_{\left(\tau_{H} \leq T\right)}\right], H<K .
\end{aligned}
$$

The down and out call option:

$$
\begin{array}{r}
C\left(S_{0}, 0\right)=e^{-r T} \sum_{i=0}^{n} \sum_{j=n-i}^{n} \frac{n !}{i ! j !(n-i-j) !} P_{u}^{i} P_{m}^{j} P_{d}^{n-i-j}\left[\left(S_{T}-K\right)^{+} \circ I_{\left(\tau_{H}>T\right)}\right. \\
\left.-S_{0} u^{i} 1^{j} d^{n-i-j}\right], H>K .
\end{array}
$$


The down and out put option:

$$
\begin{aligned}
C\left(S_{0}, 0\right)=e^{-r T} \sum_{i=0}^{n} \sum_{j=n-i}^{n} \frac{n !}{i ! j !(n-i-j) !} & P_{u}^{i} P_{m}^{j} P_{d}^{n-i-j}\left[S_{0} u^{i} 1^{j} d^{n-i-j}\right. \\
& \left.-\left(S_{T}-K\right)^{+} \circ I_{\left(\tau_{H}>T\right)}\right], H>K .
\end{aligned}
$$

\section{Conclusion Analysis}

Some scholars has researched the barrier option by the trinomial model and the listed data can show the accuracy of this method. What's more, the convergence of the trinomial model has been proved again. But the analytic expression of the barrier option have not been gained, in this paper we reached this goal. Like the other exotic options' pricing model under the trinomial tree model, we have concluded the pricing model of the barrier options under the trinomial tree model. Theoretically, it has a better effect than the binary tree model. We take the up and in call option into consideration, and analogize the other 8 kinds of barrier options' pricing model. So it can be an efficient way to pricing the barrier options.

\section{References}

[1] Gong Wen Xiu and Gao Ling Yun, The pricing model of the compound options under the trinomial tree, Statistic and Decision 18 (2016), 83-86.

[2] You Haixing and Gao Jun, The comparison study of the up and in barrier option under the binary tree or Monte-Carlo method, Modern Business 20 (2014), 168-169.

[3] Ma Lian, The Pricing Model of the Rainbow Barrier Option, Central China Normal University, 2013.

[4] Xiong Jun, The Pricing Model of the Look Back Option Under the Trinomial Tree Model, Yangzhou University, 2013.

[5] Wang Yang, Zhang Ji Zhou and Fu Yi, The pricing model of the double barrier option, Journal of Shanghai Normal University: Natural Science Edition 38(04) (2009), 347-354.

[6] Zhang Xiang Wen, The Study of the Barrier Option, Xiamen University, 2007. 
[7] Ding Zheng Zhong and Zeng Hui, The pricing model of the real option under the trinomial tree, Statistical Research Journal 11 (2005), 25-28.

[8] Peter Ritchken, On pricing barrier options, The Journal of Derivatives Winter 3(2) (1995), 19-28.

DOI: https://doi.org/10.3905/jod.1995.407939

[9] L. C. G. Rogers and O Zane, Valuing moving barrier options, Journal of Computational Finance 1(1) (1997), 5-11

DOI: https://doi.org/10.21314/CF.1997.003 